DE9 $0 \quad 012407$

\title{
Some Remarks Concerning My Research Iri Number Theory
}

\author{
R.M. Sternheimer \\ Department of Physics \\ Brookhaven National Laboratory \\ Upton, New York 11973
}

\section{DISCLAIMER}

This report was prepared as an account of work sponsored by an agency of the United States Government. Neither the United States Government nor any agency thereof, nor any of their employees, makes any warranty, express or implied, or assumes any legal liability or responsibility for the accuracy, completeness, or usefuloess of any information, apparatus, product, or process disclosed, or represents that its use would not infringe privately owned rights. Reference herein to any specific commercial product, process, or service by trade name, trademark, manufacturer, or otherwise does not necessarily constitute or imply its endorsement, recommendation, or favoring by the United States Government or any agency thereof. The views and opinions of authors expressed herein do not necessarily state or reflect those of the United States Government or any agency thereor.

This manuscript has been authored under contract number DE-AC02-76CH00016 with the U.S. Department of Energy. Accordingly, the U.S. Government retains a non-exclusive, royalty-free license to publish or reproduce the published form of this contribution, or allow others to do so, for U.S. Government purposes. 
The purpose of the present paper is to describe in some detail how I came to be interested in certain problems in number theory, while my career has been that of a theoretical physicist, specializing in atomic physics, although I have also done extensive work in other branches of physics, e.g. high-energy or particle physics. In my opinion (perhaps shared by others), my main contributions to atomic physics are: (1) the discovery and extensive calculations of the Sternheimer shielding and antishielding factors $R$ and $y_{\infty}$ for the quadrupole hyperfine structure of ions and atomic states (1949-1986), and (2) my derivation and extensive calculations of the density affect term $\delta$ for the ionization energy loss of relativistic charged particles in matter (1946-1984).

Concerning topic (1): fur an overview and a historical account of the subject of Sternheimer shielding and antishielding factors for both nuclear quadrupole and hexadecapole moments, and related effects, such as the dipole and quadrupole polarizabilities $\left(\alpha_{d}\right.$ and $\left.\alpha_{q}\right)$, and the dipole shielding factors $\xi$, I refer the reader to my detailed review article for the 8th NQR Symposium which was held in Darmstadt, Germany in July 1985; see Ref. 1. Concerning topic (2), I refer the reader both to my first paper on the density effect (1952) (see Ref. 2) and also the most recent paper on the subject by Stemheimer, Berger, and Seltzer (1984), which also contains an extensive list of references to my earlier works (see Ref. 3).

I will now proceed to a discussion and historical account of my research in number theory. My direct interest in problems in number theory started on a day in early 1960 (probably January or February) when I was still working at the BNL Cosmotron, and Hartland Snyder (who had just retumed from a trip to California) came into my office and told me. "You know, Feynman told me about an interesting function", namely:

$$
f(x)=x^{x^{x^{x^{x^{. \cdot x}}}}}
$$


while $\mathrm{x}$ is real and positive, and the number of $\mathrm{x}$ 's is infinite. This function was first considered and published by L. Euler in 1748 [4]. In Eq. (1), it is implied that the order of taking the powers is from "the top down to the bottom" of the ladder. To make this point clear, suppose that I define $f_{3}(X)=x^{x^{x}}$, with three $x$ 's only. Then I mean: $f_{3}(x)=x^{\left(x^{x}\right)}$

As an example, for $x=3, f_{3}(3)=3^{\left(3^{3)}\right.}=3^{27}=7.6256 \times 10^{12}, \quad$ and not $\left(3^{3}\right)^{3}=3^{9}=19,683$, In other words, the operation of iterated exponentiation, as shown in Eqs. (1) and (2), is non-associative, a point to which I shall return later. Concerning, $n$, the ratio between the two results is $3^{18}=3.874 \times 10^{8}$, certainly a large number!

A partial solution of Eq.(1) is obtained by solving the equation:

$$
\mathbf{x}^{\mathrm{f}}=\mathbf{f}
$$

which + arises directly from the definition of $f(x)$ including the requirement that the exponentiations will be carried out from the top to the bottom of the ladder (in that order).

It can be rather easily shown that the maximum value of $x$ for which $\mathrm{Eq}$. (3) can be satisfied is for $x_{0}=e^{1 / e}=1.444,668 \ldots$ In this case $f\left(x_{0}\right)=e$. However, we can consider smaller values of $x$ (in the region $1<x<x_{0}$ ), and we then obtain two solutions for $f$. As an example, for $x=\sqrt{2}$, we have two solutions for $f$, namely $f_{1}=2$ and $f_{2}=4$. In order to prove this, we note that $\left(\sqrt{2}^{2}=2\right.$ and $(\sqrt{2})^{4}=4$, both of which satisfy Eq. (3).

When $x$ lies in the region $e^{-e}<x<1$, then Eq. (3) has only a single solution. This matter and the importance of the value $x=e^{-e}=1 / 15.1542=0.065988$ will be discussed below.

At that time (i.e. in 1960), I calculated values of $x$ from the equation:

$$
\ln x=\frac{\ln f}{f},
$$

which I derived by taking the logarithm of both sides of (3), but I did not obtain any further interesting results. 
As I have discussed in a recent paper (Ref. 5), in the Spring of 1960, I noticed that for some days of the calendar, if $M$ denotes the month $(M=1,2, \ldots 12)$ and $D$ denotes the day ( $D=1,2, \ldots 28-31$, depending on the particular month considered), there is the relation

$$
\mathrm{MD}=\mathrm{Y} \text {, }
$$

where $\mathrm{Y}$ is the year, denoted by just the last two digits, e.g; $Y=60$ for 1960 . My attention was drawn to this fact because I usually wrote the date at the top of every page of my physics calculations. Now, for March 20, 1960, April 15, 1960, and May 12, 1960. written as $3 / 20 / 60,4 / 15 / 60$, and $5 / 12 / 60$, respectively, it was obvious that Eq. (5) is satisfied in all three cases. I called these days "Product-days". In fact, there were 3 additional product-days for 1960, namely 6/10,10/6, and 12/5. In subsequent years (in fact from 1960 to 1988), whenever I mentioned "product-days", e.g., 5/13/65 in 1965, I was usually ridiculed. As a result, I was discouraged from carrying out a systematic calculation, and from obtaining a list of all product-days in any given century.

Finally, in January 1989, I did make a listing of all product-days in a century. I found $\sum \mathrm{n}_{\mathrm{pD}}=211$, but my friend Michael Creutz put the problem on his IBM-PC Computer, and gave me a listing which showed that there are 212 product-days in a century. I was grateful for this correction. I had inadvertently left out $3 / 26 / 78$. I wrote up my results in February 1989, and also obtained a listing of all "Sum-days", which I defined as the days such that:

$$
M+D=Y \text {. }
$$

It may be noted that the maximum possible value of $\mathrm{Y}$ for Eq. (6) to be satisfied is $Y=43$, corresponding to December 31,1943 . Obviously $M+D$ cannot exceed $31+12=43$. The resulting paper has been recently published in the Joumal of Recreational Mathematics (JRM) in Vol. 22, 39-51 (1990) [Ref.5].

At this point, I should mention that in accepting this paper (in February 1989), Dr. Joseph S. Madachy, the Editor of JRM asked me whether I had also considered "product- 
years" or more accurately "product-year days" (PY-Days), i.e. days such that the complete year $Y_{c}$ (all digits) can be written as:

$$
\mathbf{Y}_{\mathrm{c}}=\mathrm{MDx} \text {, }
$$

and $x$ is the excess of $Y_{c}$ over the corresponding century year, $100 \mathrm{n}$, or, to put it more simply, $x$ represents the last 2 digits of the complete year $Y_{c}$. Thus, $x=Y$ of the above discussion. Dr. Madachy suggested two examples, both pertaining to the year $Y_{c}=1995$, namely $3 / 7 / 95$ and $7 / 3 / 95$, since $7 \times 3 \times 95=1995$. Actually, ari obvious third case for $Y_{c}=1995$ is $1 / 21 / 95$. In fact, all days with the same value of MD (for a given year $Y_{c}$ ) will satisfy Eq. (7), if this equation is satisfied for any one of these days.

Therefore, I proceeded to obtain all of the product-year days (PY-Days) from the year 100 A.D. to the maximum possible year $Y_{c}$, which turns out to be $Y_{c}=26,880$ A.D.

The corresponding day is $12 / 28 / 26,880$. (It is easily verified that $12 \times 28 \times 80=26,880$.)

In view of the rather stringent conditions imposed on M,D, and $x$ in Eq. (7), I believed at first that the number of PY-days, or as I have also called them "complete product days" will be very rare. This was found not to be the case. As an example, for the 20th Century, there are 13 complete product-days, namely $4 / 24 / 1920,6 / 16 / 1920,8 /$ $12 / 1920,12 / 8 / 1920,7 / 11 / 1925,11 / 7 / 1925,3 / 17 / 1938,3 / 13 / 1950,1 / 26 /$ 1976, 2/13/1976, 1/21/1995, 3/7/1995, and 7/3/1995. These 13 Py-days perrain to 6 product-years, namely 1920, 1925, 1938, 1950, 1976, and 1995. The average ratio $\Sigma N_{P Y-D} / \sum N_{P Y}$ is therefore $13 / 6=2.17$ for the years from 1900 to 2000 A.D.

The number of PY-days per century decreases with increasing $Y_{c}$. This is reflected in the fact that although there are 13 PY-days for the 20th century, there are only 15 PYdays fo: the range of $Y_{c}$ from 20,000 to 27,000 , i.e., $15 / 70=0.214$ per century, on the average. The paper which contains these results will be published in JRM, vol $\underline{22}$ (1990) [see Ref. 6].

Now, returning to the period of the early 1960 's: In 1962, I obtained another interesting result. About the time of my 36th birthday on April 26, 1962, I asked myself 
how much longer I would have to live if I want to become 100 years old. This is almost certainly an academic question, but the answer is, of course, 64. Then I noticed that $36=6^{2}$ and $64=2^{6}$, and I calculated about 100 numbers of the form.

$$
g(x, y) \equiv x^{y}+y^{x},
$$

where $\mathrm{x}$ and $\mathrm{y}$ are positive integers. At first, I was interested as to whether any of these numbers $g(x, y)$ (with reasonably small values of the integers $x$ and $y$ ) would also be a power of 10 , in the same manner as $g(2,6)=2^{6}+6^{2}=100$. The answer was negative.

Nevertheless I looked up a textbook on number theory (probably by G.H. Hardy) and I found that most (if not all) of the researches conducted for equations like Eq. (8) were concerned with functions of the form:

$$
g_{n}(x, y)=x^{n}+y^{n} ;
$$

they were homogeneous forms related to Fermat's Last Theorem (FLT). Obviously Eq.(9) is the appropriate equation for FLT if $g_{n} \equiv z^{n}$.

About fifteen years later, in fact in 1977, after considering Eq. (8) and because I am a theoretical physicist and am naturally familiar with quantum mechanics, I realized that Eq. (8) represents a symmetric combination of $x^{y}$ and $y^{x}$, because of the $+\operatorname{sign}$ in the right-hand side. Therefore, in analogy to the quantum-mechanical wave function, I calculated values of the function $f(x, y)$ defined as:

$$
f(x, y)=x^{y}-y^{x} \text {, }
$$

which is antisymmetric in $x$ and $y$. I calculated many values of $f(x, y)$ for reasonably small values of the integers $x$ and $y$. A table of these results is presented in Appendix A (by myself) in a BNL Informal Report, BNL-23308, by M. Creutz and R.M. Sternheimer (September 1977). (Ref. 7). I obtained five results which were particularly interesting to me:

$$
\begin{gathered}
f(2,5)=2^{5}-5^{2}=+7 \\
f(2,4)=2^{4}-4^{2}=0 \\
f(2,3)=2^{3}-3^{2}=-1
\end{gathered}
$$




$$
\begin{aligned}
& f(1, y)=1^{y}-y^{1}=1-y, \\
& f(0, y)=0^{y}-y^{o}=-1 .
\end{aligned}
$$

(Note that only positive integers and 0 are considered for $\mathrm{x}$ and $\mathrm{y}$.)

I later on focused on the result of Eq. (12), particularly in connection with the work of Ref.10 and this gave me the inspiration for a new conjecture concerning Fermat's Last Theorem. This conjecture which I found in September 1983 will be discussed below.

I will now return to the discussion in a chronological order. Between 1962 and 1977, i.e., for 15 years, I was very busy with my physics research, both in connection with calculations of the various Sternheimer shielding and antishielding factors, for nuclear quadrupole moments and nuclear hexadecapole moments [see Ref. 1], and also with a study of regularities of the energy levels of the various electrons for atoms and ions throughout the Periodic Table. I have called this phenomenon "the $\mathrm{k}$-ordering" of atomic and ionic energy levels. I wrote a review article on the k-ordering in 1980; it is published in Ref. 8.

However, around 1976, I had several conversations with Bruce Martin who was then the BNL member of the Fortran Updating Committee. On June 8, 1977 (Wednesday), I had dinner with B. Martin at the Brookhaven Center, and sometime during our discussion, I had a "brainstorm". First, I remembered the details of my conversation with H. Snyder about the function $f(x)$ [Eq. (1)] in early 1960, i.e. more than 17 years earlier. Then I realized that one of the most important properties of $f(x)$ of Eq. (1) is that it is non-associative, i.e., the order of the various exponentiations must be strictly specified, as I have discussed above. Then I thought of an important generalization of $f(x)$, namely $(x)^{3}$ which $i$ defined as follows:

$$
F_{n}(x)
$$




$$
F_{n}(x) \equiv g_{1}(x)^{g_{2}(x)^{g_{g}(x)}},
$$

where the $g_{j}(x)$ are real positive functions of $x$. In fact, as a short-hand notation for $F_{n}(x)$, I also invented the simple notation.

$\sqsupseteq \quad F_{n}(x)=\sum_{j=1}^{n} g_{j}(x)$,

where I picked the symbol (cap "ksi") in analogy to $\Sigma$ for sum and $\Pi$ for product.

The function $F_{n}(x)$ has several interesting properties when $g f(x)$ decreases with increasing $j$, for any fixed value of $x$. As an example, I will choose;

$$
g_{j}(x) \equiv \frac{x}{j} \text {. }
$$

Now I consider an integer $x$, say $x=3$. Then the exponentials involved in $F_{n}(x)$ for large $\mathrm{n}$, essentially terminate at $\mathrm{j}=3$. To see this, I write down as an example:

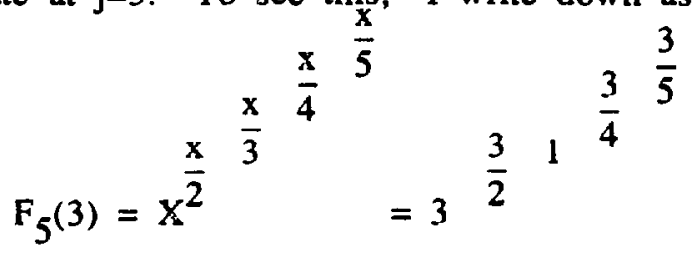

Now it is obvious that the integer 1 raised to any finite power is simply equal to 1 .

Therefore,

$$
F_{5}(3)=F_{4}(3)=F_{3}(3)=3^{3 / 2}=5.196
$$

A second illustrative example is:

$$
g_{j}(x) \equiv \frac{x}{j^{2}} .
$$

In this case, a simple result will be obtained if we use a value of $x$ which is the square of an integer, e.g., $x=4$. This gives

$$
F_{3}(4)=\left(\frac{4}{1}\right)^{(4 / 4)^{(4 / 9)}}=4^{1}=4 .
$$


In the same month, namely June 1977, I wrote a Brookhaven Informal Report, BNL-23081, entitled "On a Set of Non-Associative Functions of a Single Positive Real Variable" (Ref. 9). Michael Creutz received a copy of this paper, and was interested in further properties of the function $f(x)$ and the various possible functions $F_{n}(x)$ which I had defined in Ref. 9.

The details of the studies of $f(x)$ and of various $F_{n}(x)$ are presented in a paper of Creutz and Sternheimer (Ref. 10) and also in the aforementioned more detailed report, i.e. Ref. 7. In particular, we have discovered a property which I have called "dual convergence". As an example, for $f(x)$ with $x<e^{-e}=0.065988$, the function $f(x)$ of Eq. (1) is dual-convergent, in the sense that if we consider an odd number of $x^{*}$, e.g., $x^{x^{x}}$, the resulting functions, namely

$$
f_{1}(x)=x, \quad f_{3}(x)=x^{x^{x}}, f_{5}(x)=x^{x^{x^{x^{x}}}} \text { etc., }
$$

will converge to one value of $f$, namely $f_{\text {odd }}(x)$, which for $x=0$, is zero, whereas the functions $f_{n}(x)$ with $n=e v e n$, such as $f_{2}(x)=x^{x}, f_{4}(x)=x^{x^{x}}$, will converge to another (larger) value, namely $f_{\text {even }}(x)$, which for $x=0$ is 1 . The same phenomenon occurs for the more general functions $F_{n}(x)$ [see Eq. (16)], as is shown for example in Fig. 3 of our paper of Ref. 10, with $F_{10}(x)$ for $g_{j}(x)=\frac{x}{j^{2}}$ [cf. Eq. (21) above] and $F_{11}(x)$ for the same $g_{j}=\frac{x}{j^{2}}$. Thus for $x=1,4,9$, we obtain a unique value of $F_{n}(x)$, since each $x$ is the square of an integer. We find $F_{d}(1)=1, F_{n}(4)=4$, and $F_{n}(9)=9^{9 / 4}=140.2961$, regardless of whether $\mathrm{n}$ is even or odd, as has been discussed above.

However for non-integral values of $x$, e.g. for $x<1$ and for $1<x<4$, the functions $F_{10}\left(g_{j}=x / j^{2}\right)$ and $F_{11}\left(g_{j}=\frac{x}{j^{2}}\right)$ differ to some extent, and obviously not as much for the interval $1<x<4$ as for $x<1$. In the latter case, the two curves for $n=10$ and $n=11$ differ by a relatively large amount. In fact for $x=0, F_{11}=0$, whereas $F_{10}=1$, in the same manner as for the simple function $f(x)$, i.e., the functions $f_{\text {odd }}(x)$ and $f_{\text {even }}(x)$, as discussed above [see Eq. (23)]. 
In the paper of Ref. 11, we have discussed in detail a particular case of the function $F_{n}(x)$ [Eq. (17)], which I had introduced in June 1977 (Ref. 9), namely the case where $g_{5} \equiv x$ for $j=o d d$, and $g_{j} \equiv y$ for $j=e v e n$. This leads to the following function:

$$
F\left(x_{1} y\right)=x^{y^{y^{\circ}}}
$$

where the ladder on the right hand side is infinite, i.e. it has an infinite number of $x$ 's and $y$ 's. Both $x$ and $y$ are assumed to be real and positive, as in the preceding discussion. Obviously $F(x, y)$ is considerably more complicated than $f(x)$, since it depends on two positive real variables $x$ and $y$ instead of one variable only $(x)$. In the course of the work of this paper [11], I noticed that a point of tangency occurs in Fig. 3 of Ref. 11 at a value of $y$ very close to 15 . A few days later, 1 realized that the accurate location of the tangency point is given by

$$
y=e^{e}=15.154261,
$$

i.e., just the reciprocal of the value $x_{0}=e^{-e}=0.0659880$, for which $f(x)$ becomes dualconvergent (i.e., for $x<x_{0}$ ). Moreover, the ${ }_{A}^{*}$ of the limi of convergence for $f(x)$, namely,

$$
x=x_{1}=e^{1 / e}=1.444,668,
$$

equals $e^{-1 / e}=0.692201$, and this is the value of $x$ for the tangency point in Fig. 3 of Ref. 11.

A function which is obtained by repeated roots of $x$ (in a real sense, the obverse of the function $f(x)$ of Refs. 7 and $\stackrel{10}{f}$ ) was discussed by Creutz and Sternheimer in Ref. 12 (submitted in May 1979). This "reciprocal function" has been denoted by $\phi(x)$ and is defined as follows: 


$$
\begin{aligned}
& \sqrt{x} \sqrt{x} \\
& \sqrt{x} \\
& \phi(x) \equiv \quad \sqrt{\mathbf{x}} .
\end{aligned}
$$

It can be shown that

$$
\phi(x)=\sqrt[\phi(x)]{x}
$$

provided that the sequence of Eq. (27) has a nontrivial limit. Equation (28) leads to the following equation:

$$
\phi(\mathbf{x})^{\phi(x)}=\mathbf{x}
$$

Equations (28) and (29) plus the equation for $f(x)$, namely Eq. (3), lead to the following useful relation.

$$
\phi(x)=\frac{1}{\mathrm{f}(1 / \mathrm{x})} .
$$

Thus because $f(\zeta)$ is dual-convergent for $\zeta<e^{-e}, \phi(x)$ is dual-convergent for $x>e^{e}$. This property is clearly shown in Fig. 1 of Ref. 12. Moreover, $\phi(x)=0$ for $x<e^{-i / e}=$ 0.69220 , corresponding to the fact that $f(x)$ is infinite for $x>e^{1 / e}=1.444668$.

I shall now return to Fig. 1 of Ref. 10 . This figure gives the values of $\mathbf{x}$ as a function of $f$ for which the equation

$$
\mathrm{x}^{\mathrm{f}}=\mathrm{f}
$$

is satisfied. At this point, $I$ will include the second branch of the curve of $x$ us $f(x)$, the branch for which $f(x)>e$. Although this branch is not relevant to the study of $f(x)$ as defined above [see Eq. (1)], it is nevertheless useful in the following context. Obviously for $0 \leq x \leq \mathrm{e}^{1 / \mathrm{e}}=1.444,668$, this curve shows that there are two solutions for $\mathrm{f}$ for a given $x$ lying in the aforementioned range. Thus we have

$$
\mathrm{x}^{\mathrm{f}}=\mathrm{f}_{1}, \quad \mathrm{x}^{2}=\mathrm{f}_{2},
$$

or equivalently 


$$
\mathrm{x}=\mathrm{f}_{1}{ }^{\mathrm{l} / \mathrm{f}} 1=\mathrm{f}_{2}{ }^{1 / \mathrm{f}_{2}}
$$

As is shown by Fig. 1 of Ref.10, one of the $f$ 's, say $f_{1}$, is smaller than e, and the other $f$, namely $f_{2}$ is larger than e. If we require that $f_{1}$ be an integer, we have just two integers $<e$, namely $f_{1}=1$ and $f_{1}=2$. We first consider the case of $f_{1}=2$; this corresponds to $x=\sqrt{2}$.

The two solutions of $f_{1}$ and $f_{2}$ are then: $f_{1}=2, f_{2}:=4$, since:

$$
\sqrt{2}=2^{1 / 2}=4^{1 / 4} \text {. }
$$

In fact, another way of writing Eq. (33) is obviously:

$$
\mathrm{f}_{1} \mathrm{f}_{2}=\mathrm{f}_{2} \mathrm{f}_{1} \text {. }
$$

and if we change the variables $f_{1}$ to $x_{\lambda}$ and $f_{2}$ to $y$ [obviously not the $x$ used in Eq. (33)], Eq. (35) becomes

$$
x^{y}=y^{x} \text {, }
$$

or: $x^{y}-y^{x}=0$. Obviously for $x=2, y=4, x^{y}=y^{x}=16$, fsomewhat above the minimum possible value which is attained for $f_{1}=f_{2}=e$, namely $f_{1} f_{\mathcal{L}}=f_{2} f_{1}=e^{e}=15.154261$.

Whereas the solution of $(33)$ for $f_{1}=2$ is obvious (namely $f_{2}=4$ ), it is also possible to show that as $f_{1}$ approaches 1 from above (i.e., from $1+\varepsilon$ ), $f_{2}$ becomes infinite in a wellcontrolled and unique way. As an example, for $\hat{\mathrm{r}}_{1}=1.0 \mathrm{I}$, we find $\mathrm{f}_{2}=658.8$, since

$$
1.01^{658.8}=658.8^{1.01}=702.96 \text {. }
$$

Sometime after this observation, (actually on September 23, 1983), I started to think about Equation (36) in connection with an analogy to Fermat's Last Theoreum (FLT).

Note that $y$ is clearly a well-defined function of $\mathbf{x}$ in Eq. (36), and the only possible integer values of $x$ are $x=1$ and 2 . On the other hand, the equation for Fermat's Last Theorem namely:

$$
x^{n}+y^{n}=z^{n},
$$

when $n$ is an integer has solutions only for $n=1$ and $n=2$. I have shown this above for Eq. (36). For Eq. (38), the situation is identical, namely $\mathrm{n}$ can only have the values $I$ and 2 .

Thus we have the familiar Pythagorean triplets ( $x, y, z=$ integers and $n=2$ ) for the equation

$$
x^{2}+y^{2}=z^{2}
$$


The second case, namely $n=1$,

$$
x+y=z,
$$

may appear trivial, but it is nevertheless obviously a solution of Fermat's equation (38). In fact, if $z$ is even and we assume that $x>y$, and both $x$ and $y$ are positive integers, then there are $z / 2$ solutions of Eq. (40), whereas for $z=o d d$, the number of solutions is $\frac{z-1}{2}$.

It is of interest that for $n=0$, Eq. (38) has no solution since $x^{\circ}+y^{\circ}=2$, whereas $z^{o}$ on the right-hand side equals 1.

I wrote up this conjecture about Fermat's Last Theorem in September 1983 and it was published in Ref. 13. Specifically the conjecture states that Equation (38) can be satisfied only for the two positive integers 1 and 2 , which are smaller than e. So far as I know, Eq. (40) has never previously been regarded as being connected with Fermat's Last Theorem, even though $n=1$ is obviously a positive integer. Perhaps a different way of stating my point of view is that FLT makes a statement about the following function F of four integers: $x, y, x$, and $n$, namely:

$$
F(x, y, z ; n) \equiv z^{n}-x^{n}-y^{n}=0 .
$$

In the paper of Ref. 13, I also presented and proved a useful formula for

$$
f(x, y)=x^{y}-y^{x} \text {; }
$$

[of Eq. (10)] when $x$ and $y$ differ by a small amount $\Delta$. The result is

$$
f(x, y) \equiv x^{x}(y-x)(\ln x-1) \text {. }
$$

This equation shows at once that for $y=x+\Delta$, if $\ln x>1$, i.e., $x>e$, then $f(x, y)$ [Eq. (43)] will be positive provided that $y>x$, i.e., $\Delta>0$. For $x<e$, the converse is true. As an example, $3.5^{3.6 .6}=90.92$ is somewhat larger than $3.6^{3.5}=88.52$. Incidentally, I had obtained the result (43) six years earlier, in 1977, and the proof was given in Appendix A of Ref. 7.

I may also note that just as $F(x, y, x ; n)$ has no solution for $n=0$, so analogously the equation

$$
f(x, y)=x^{y}-y^{x}=0,
$$


also has no solution when either $x$ or $y=0$. Thus for $x=0, f\left(0, y \bar{x}_{n}{ }_{-1}{ }^{\circ}=-1\right.$ for all positive real $y$.

After my work of Ref. 13 was completed, I realized that if $n<e$ was indeed the determining condition for the validity (and the proof) of FLT, then a thorough study of the case $n=2$ (the largest integer smaller than e) would be worthwhile, because it might shed some light on the relevance of e to the proof of FLT. This led me to carry out a systematic study of the Pythagorean triplets $x, y, z$, such that

$$
\mathrm{x}_{\mathrm{i}}^{2}+\mathrm{y}_{\mathrm{i}}^{2}=\mathrm{z}^{2}
$$

It turned out that this study led to a number of interesting results, including in particular the general expression for the number $n_{d}$ of Pythagorean decompositions of an arbitrary integer z. The results are contained in three papers which I will now describe: (19ej, 14, 16, 17),

In the first (and most detailed) paper (Ref. 14), I considered first all Pythagorean decompositions of the integers $z$ from 1 to 500. Obviously many integers $z$ do not have a Pythagorean decomposition, (P-decomposition) whereas other integers $\mathrm{z}$ have one or more P-decompositions. Dr. Michael Creutz kindly devised a computer program to list all P. decompositions in the range $1<z<500$. At that time (October 1983), we used a large H:P. Computer (it occupied about half the size of a desk top); the entire calculations took about 10-15 minutes. In the resulting computer output, I separated the primitive solutions of $z^{2}=x^{2}+y^{2}$ from the non-primitive solutions, for which $x, y$, and $z$ are not relatively prime. Obviously an example of a primitive solution is $(x, y, z)=(3,4,5)$, whereas $(x, y, z)=(15,20,25)$ is not a primitive solution, since $x, y, z$ have the common factor 5 .

The main interest concems the primitive solutions of the type of $(x, y, z)=(3,4,5)$, $(12,5,13),(15,8,17)$, etc. In a second calculation, Creutz obtained a list of the primitive solutions only for the integers $z_{i}$ from 1 to 3000 . In Ref. 14, I have also defined two types of prime numbers, namely the P primes (for "Pythagorean primes") denoted by $P_{t}$, such that $p_{t} \equiv 1(\bmod 4)$, and the NP primes (non-P primes) denoted by $g_{j}$, for which $q_{j} \equiv 3(\bmod 4)$.

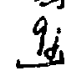


Thus the set of all $p_{i}$ and all $q_{j}$ plus the integer 2, consititutes the set of all prime numbers. Obviously any arbritary integer $N_{k}$ can be written as follows:

$$
N_{k}=\left(\prod_{i=1}^{n_{a}} p_{i}^{\alpha_{i}}\right)\left({ }_{2}^{\beta} \prod_{j=1}^{n_{b}} q_{j}^{\beta_{j}}\right) \equiv A_{k} B_{k},
$$

where the $\alpha_{l}, \quad \beta$ and the $\beta_{j}$ are positive integer exponents, and $A_{k}, B_{k}$ denote the first and the second prociuct on the right-hand side, respectively; $\mathbf{n}_{\mathrm{a}}$ is the total number of different $P$ primes, $P_{i}$ involved, and similarly $n_{b}$ is the total number of different NP primes $q_{j}$ in the prime decomposition of $N_{k}$.

In Ref. 14, I have obtained two equivalent expressions for the number $n_{d}$ of $P$ decompositions of an arbitrary positive integer $N_{k}$. (Note that $n_{d}$ includes both the primitive and the non-primitive decompositions of $N_{k}$.) The first formula for $n_{d}$ is given by Eq. (13) of Ref. ${ }_{n} 14$, namely

$$
n_{d}=\sum_{i=1}^{n_{a}} \alpha_{i}+2 \sum_{\alpha<j} \alpha_{i} \alpha_{j}+4 \sum_{i<j<k}^{n_{k}} \alpha_{i} \alpha_{j} \alpha_{k}+\ldots+2^{n_{a}-1} \alpha_{1} \alpha_{2} \alpha_{3} \ldots \alpha_{n_{a}}
$$

Obviously $n_{d}$ depends only on the first product of $N_{k}$, namely $A_{k}=\prod_{i=1}^{n_{a}} P_{i} \alpha_{i}$ [see Eq. (46)] and in fact it depends only on the set of the exponents $\alpha_{1}$.

As an example of Eq. (47), $n_{d}(125)$, i.e., $n_{d}$ for $N_{k}=125$ is 3 , since $125=5^{3}$, so that $\alpha_{1}=3$ in Eq. (47), and all other $\alpha$ 's are zero. Similarly

$$
n_{d}(65)=n_{d}(5 \times 13)=1+1+2(1)(1)=4 \text {, }
$$

because both 5 and 13 have the exponent 1 , and thus $\alpha_{1}=\alpha_{2}=1$ in Eq. (46). In fact, the integer 65 is the smallest integer for which $\alpha_{1}=\alpha_{2}=1$ and $n_{d}=4$. (Obviously both 5 and 13 are $\equiv 1(\bmod 4)$ and are therefore P primes.) Two more complicated results will be cited:

$$
n_{d}(325)=n_{d}\left(5^{2} \times 13\right)=2+1+2(2)(1)=7,
$$

since $\alpha_{1}=2$, and $\alpha_{2}=1$ in Eq. (47). In addition, the second example is $N_{k}=1105=5 \times 13 \times 17$, so that $\alpha_{1}=\alpha_{2}=\alpha_{3}=1$. Equation (47) then gives:

$$
n_{d}(1105)=3+2[(1)(1)+(1)(1)+(1)(1)]+4(1)(1)(1)(1)=3+6+4=13 \text {. }
$$


It is obvious from these calculations that when more than three $\alpha_{i}$ 's are involved, the evaluation of Eq. (47) becomes rapidly more complex and more lengthy. For this reason, I have derived an alternative and much simpler formula for $n_{d}$ in Appendix I of Ref. 14. The result is:

$$
n_{d}\left[\alpha_{i}\right]=(1 / 2) \prod_{s=1}^{s_{\max }}\left(2 k_{s}+1\right)^{\gamma_{s}}-1 / 2 \text {, }
$$

[see Eq. (A25) of Ref. 14]. The set [ $\alpha_{i}$ ] is the set of all of the values of $\alpha_{i}$ in Eq. (46), i.e., the set of all the exponents of the $P$ primes $p_{i}$ in Eq. (46), [the primes $p_{i} \#(\bmod 4)$ ]. In Eq. (51), $\mathrm{k}_{\mathrm{s}}$ is a particular value of $\alpha_{i}$ and $\gamma_{s}$ is the number of times this value of $\alpha_{i}$ occurs in the decomposition of $N_{k}$. Thus the subscript $s$ identifies the different values of $\alpha_{i}$, and we find

$$
\sum_{s=1}^{s_{\text {mix }}} \gamma_{s}=n_{3}, y_{x_{8}}^{s}=\ln _{\mathrm{a}}
$$

where $n_{a}$ is the total number of different $p_{\mathfrak{t}}$ primes which occur in the prime decomposition of $N_{k}$. For the preceding three examples [Eqs. (48), (49) and (50)], we obtain

$$
n_{d}(65)=(1 / 2)\left(3^{2}-1\right)=4,
$$

since $2 \alpha_{i}+1=3$ and $\alpha_{1}=1$ occurs iwice. Also

$$
\mathbf{n}_{\mathrm{d}}(325)=(1 / 2\lfloor(3)(5)-1]=7,
$$

since $\alpha_{1}=2$ and $\alpha_{2}=1$ both occur once; and $2 \alpha_{1}+1=5$ and $2 \alpha_{2}+1=3$. Finally, concerning Eq. (50) for $N_{k}=1105=5 \times 13 \times 17$, the exponent $\alpha_{1}=1$ occurs 3 times giving $2 \alpha_{k}+1=3$ and $\gamma_{1}=3$, so that

$$
n_{d}(1105)=(1 / 2)\left(3^{3}-1\right)=13 \text {. }
$$

Finally, I consider a much larger integer, namely $N_{k}=22,100$. We have

$$
22,100=2^{2} \times \underline{5}^{2} \times \underline{13} \times \underline{17},
$$

where I have underlined the P primes $\underline{5}, \underline{13}$ and $\underline{17}$. The factor 5 has the exponent $\alpha_{1}=2=k_{1}$, 5 Therefore it contributes a factor $2 k_{1}+1=5$ in Eq. (51). The factors 13 and 17 have exponents $\alpha_{2}=\alpha_{3}=1$, giving $2 k_{2}+1=3$, and because there are two such factors we take $\gamma_{2}=2$, and as a result the contribution to the product in Eq. (51) is $3^{2}$. Thus altogether we find: 


$$
n_{d}(22,100)=(1 / 2)\left[5.3^{2}-1\right]=22 .
$$

I may note that the number of primitive solutions of Eq. (45) is appreciably smalles than $n_{d}$. First of all, any integer for which $B_{k}$ is not equal to 1 [see Eq. (46)], i.e., any integer which contains factors of $2^{\beta}$ or any of the $q_{j}{ }_{j}$ obviously cannot have a primitive solution, since all solutions are proportional to $B_{k}$. It has been shown by Ecken (Ref. 15) that for integers with $B_{k}=i$, the number of primitive solutions $n_{P S}$ is given by

$$
n_{p s}=2^{n} a_{-1}^{-1}
$$

where $n_{\mathfrak{a}}$ is the number of different $P$ primes $P_{\mathfrak{i}^{*}}$. Thus the individual powers $\alpha_{\mathfrak{q}}$ do not enter into the expression for $n_{p s}$. For the three integers considered above: 65,325 and 1105, we find, $n_{p s}(65)=2$ (since $\left.n_{a}=2\right), n_{p s}(325)=2$ (since again $n_{a}=2$ ), and $n_{p s}(1105)=4$ (since $n_{\alpha}=3$ ).

Besides the expressions for $\mathrm{n}_{\mathrm{d}}$ [Eqs. (47) and (51)], I obtained some additional results in Ref. 14:

(1). I obtained values of the two tunctions $f(N)$ and $g(N)$ as a function of $N$ up to $N=2000$. Here $f(N)$ is the average value of $n_{d}$ for a small interval centered on $N$, i.e., from $N-\frac{\Delta N}{2}$ to $N+\frac{\Delta N}{2}$, for those integers for which $n_{d} \geq 1$, i.e., excluding the integers for which $n_{d}=0$. This average number $\left\langle n_{d}\right\rangle=f(N)$ was found to increase rather rapidly, from $f(75)=1.36$ (for the interval from $N=50$ to 100 ) to $f(1975)=1.87$ (for the interval extending from $\mathrm{N}=1950$ to $\mathrm{N}=2000$ ).

The function $g_{-}^{-}(N)$ mentioned above is the fraction of the integers which are Pdecomposable in at least one way, i.e., for which Eq. (45) holds for at least one set of $x_{i}$ and $y_{i}$ values. This fraction increases with $N$, and in fact it was found that $g(75)=0.49$, $g(975)=0.57, g(1975)=0.61$. It is obvious that it is desirable to extend these calculations of $f(N)$ and $g(N)$ to larger $N$ values, and I have accomplished this in my paper of Ref. 16, which will be discussed below.

(2). The second set of results concerns the Goldbach Conjecture, according to which every even integer $z$ can be written as the sum of two prime numbers $x_{i}$ and $y_{t}$ in at least one way. Like Fermat's Last Theore 
to very large values of $z$, but not for all integers $z$. It seemed of interest to tabulate all of the possible combinations $x_{i}+y_{i}$ for the 50 even integers extending from $z=2$ to $z=100$. I have done this in Appendix B of Ref. 14. Two results emerge from this tabulation:

(a) Considered as a function of $z$, the number of different Goldbach decompositions $\mathrm{n}_{\mathrm{U}}$ has local maxima when $\mathrm{z}$ is divisible by 6 . As an example, for $z=84, \mathrm{n}_{\mathbf{6}}=9$, and the decompositions are as follows, $84=83+\underline{1}, 79+\underline{5}, \underline{73}+11,71+\underline{13}, 67+\underline{17}$. $\underline{61}+23, \underline{53}+31,47+\underline{37}, 43+\underline{41}$, as compared to $n_{\mathfrak{z}}=5$ for $z=82$, and $n_{\mathfrak{z}}=5$ for $z=86$.

Here I have underlined the $P$ primes $\left[p_{i}=1(\bmod 4)\right]$ in the nine decompositions of $z=84$.

(b) It is obvious both irom the table of decompositions and from general reasoning that for $z$ divisible by 2 , but not by 4 [i.e. $z \equiv 2(\bmod 4)], z$ will be a sum either of two P-primes or of two NF-primes. As an example: $82=79+3=71+11=59+23=\underline{53}+\underline{29}=\underline{41}+\underline{41}$, where the underlined integers are $P$ primes and the other integers are NP primes. On the other hand, when $z$ is divisible by $4, z=0(\bmod$ 4), as for $z=84$, obviously one of the primes must be a $P$ prime, while the other must be an NP prime, as exemplified above.

In the second paper entitled "Some Results Concerning Pythagovern Triplets II" (Ref. 16), I have extended the preceding calculations of the functions $f(N)$ and $g(N)$ from $\mathrm{N}=2000$ up to very large $\mathrm{N}$ values, i.e. up to $\mathrm{N}=10^{7}$ I have also fitted the resulting values of $f(N)$ and $g(N)$ in the range from small $N$ up to $N=10^{7}$ by means of accurate fitting functions. As would be expected, $g(N)$ increases, albeit very slowiy, to a limiting value of $g(\infty)=1$ for $N \rightarrow \infty$ Thus the function $g(N)$ as obtained from the fitting procedure up to $\mathrm{N}=10^{7}$, is given by

$$
g(N)=1-0.559 \mathrm{~N}^{-0.0475}=1-0.559 \mathrm{~N}^{-1 / 21.05} \text {, }
$$

i.e., $g(N)$ approaches 1 at large $N$ very slowly, namely approximately like the inverse 21 st root of $\mathbf{N}$.

On the other hand, the function $f(N)$ which is defined as the average number $\left\langle n_{d}\right\rangle$ of Pythagorean decompositions (for those integers for which $n_{d} \geq 1$ ) per integer is approximately given by the following expression: 


$$
f(N)=1.079 \mathrm{~N}^{0.0725}=1.079 \mathrm{~N}^{1 / 13.79} \text {. }
$$

indicating a very slow increase with $N$, i.e., approximately as the 14 th root of $N$.

Incidentally the actual values of $f_{-}^{-}\left(10^{7}\right)$ and $g\left(10^{7}\right)$ which were used in obraining Equs (59) and (60) are $f\left(10^{7}\right)=3.47$ and $g\left(10^{7}\right)=0.74$. These numberical values for $\mathrm{N}=10^{7}$ and for intermediate $\mathrm{N}$ values $\left(\mathrm{N}=22,000\right.$, and $\left.\mathrm{N}=10^{6}\right)$ were obtained by a program devised by M. Creutz which lists the prime decompositions of 500 integers centered at $N=22,000$, $\mathrm{N}=10^{6}$, and $\mathrm{N}=10^{7}$, respectively. As an example, the interval $\mathrm{N}-\frac{\Delta \mathrm{N}}{2}$ to $\mathrm{N}+\frac{\Delta \mathrm{N}}{2}$ for $\mathrm{N}=10^{7}$ extends from $10^{7}-250$ to $10^{7}+250$ (see Tables $1-3$ of Ref. 16).

I have also made a detailed analysis of the individual values of $n_{d}$ for the 501 integers extending from $\mathrm{N}_{1}=21,800$ to $\mathrm{N}_{2}=22,300$. The numbers of times $N\left(\mathrm{n}_{\mathrm{d}}\right)$ that a particular value of $n_{d}$ occurs among these 501 integers are as follows:

$$
N\left(\mathrm{n}_{\mathrm{b}}=0\right)=162, \quad N(1)=225, \quad N(2)=15, \quad N(3)=2, \quad N(4)=78, \quad N(5)=1, N(7)=9, \quad N(10)=2,
$$
$N(13)=6$ and $N(22)=1$.

Thus the number of integers for which $n_{d}>0$ is: $501-162=339$, which yiel.is

$$
g(22,000)=\frac{339}{501}=0.677 \text {. }
$$

In a similar fashion, I found that the total number of Pythagorean decompositions in this interval of $N$ is $\sum n_{d}=$, from which the average number $f(22,000)$ of $P$ decompositions for these integers (with $n_{d} \geq 1$ ) is:

$$
f(22,000)=\frac{761}{339}=2.24 \text {. }
$$

In addition, the paper of Ref. 16 contains a calculation of $h(N)$ defined as the average number of different prime numbers in the prime number decomposition of a given integer, regardless of the powers $\alpha_{i}, \beta$ and $\beta_{j}$ with which the primes appear. I found $h(1975)=2.37$ primes/integer on the average, and similarly $h(22,050)=2.61$ and $h\left(10^{7}\right)=3.12$. The resulting fit for $h(N)$ is similar to that for $f(N)$ [Eq. (60)]. The fit for $h(N)$ is given by

$$
h(N)=1.861 \cdot \mathrm{N}^{0.03205}=1.861 \mathrm{~N}^{1 / 31.20} \text {. }
$$

In connection with the frequency of the primitive solutions of the Pythagorean Equation $x^{2}+y^{2}=z^{2}, \quad I$ already noticed, from the work reported in Ref. 14, that the number 
of primitive solutions per constant interval in z, e.g., an interval $\Delta z=100$, is remarkably constant, namely $\sim 16$ primitive solutions per interval of 100 in $z$. In fact, referring to Table 1 of Ref. 14, which lists all of the primitive solutions for $1<z<500$, it is obvious that there are exactly 80 such primitive solutions in the $\Delta z=500$ interval, i.e. on the average of 16 primitive solutions per $\Delta z=100$. In fact, as is shown by Eq. (6) of Ref. 14, the total numbers of primitive solutions in the successive intervals of 100 in Table 1 are: 16 from $z=1$ to 100,16 from 101 to 200,15 from 201 to 300,16 from 301 to 400 , and 17 from 401 to 500 , giving a total of

$$
\sum \mathrm{n}_{\mathrm{PS}}=16+16+15+16+17=80 .
$$

Also the total number of the primitive solutions for the integers $\mathrm{N}$ in the entire range from 1 to 3000 is 477 , almost exactly the number expected if $n_{P S}(\Delta z=100)=16$, namely $16 \times 30=480$.

For this reason, when I extended my calculations to intervals in the regions centered on larger values of $\mathbf{N}(=z)$ in the paper of Ref. 16 ["Some Results Concerning Pythagorean Triplets. II"], I obtained the values of $N_{P S}$ for intervals $\Delta N=400$ centered on $N=22,500$, $\mathrm{N}=10^{5}, \mathrm{~N}=16^{6}$ and $10^{7}$, and the results (divided by 4 to normalize to an interval of 100 instead of 400) were again close to 16 , i.e., in the range from 14.75 to 17 for the four values of $\mathrm{N}$. These results are listed as $\mathrm{N}_{\mathrm{PS}} / 4$ in Table 1 of my recent paper of Ref. 17.

I have obtained at least a qualitative explanation for this near-constancy of $\mathrm{N}_{\mathrm{PS}} / 4$ as a function of $N(=z)$. $\quad N_{P S}$ can be written as.

$$
\mathrm{N}_{\text {PS }}=\mathrm{N}_{1}+\mathrm{N}_{2} \text {, }
$$

where $N_{1}$ is the number of powers of $P$ primes $P_{i}{ }_{i}$ in the interval, and this is expected to decrease, albeit slowly, with increasing $z$. On the other hand, $N_{2}$ is the number of primitive solutions arising from integers containing more than one $p_{i}$, i.e., ${ }_{\mathrm{z}=\mathrm{p}_{1}}{ }^{\alpha_{1}} \mathrm{p}_{2}{ }^{\alpha_{2}}, \mathrm{p}_{1}{ }^{\alpha_{1}}{ }_{\mathrm{p}_{2}}^{\alpha_{2}}{ }_{\mathrm{p}_{3}}^{\alpha_{3}}$, etc. Because of the increasing number of prime numbers $P_{i}<z$, as $s_{A}^{z}$ increases, we expect (and find) an increasing number of cases which involve more than one $p_{i}{ }_{i}$, i.e., which are of the general form 


$$
z=\prod_{i=1}^{n_{a}} p_{i}^{\alpha_{i}}
$$

It appears that the decrease of $N_{1}$ and the simultaneous increase of $N_{2}$ approximately balance each other, so that $N_{P S}=N_{1}+N_{2}$ is nearly constant (as $z$ increases) and the resulting $N_{P S} \cong 16$ for a $\Delta z=100$ interval in $z$. This possibility is well illustrated by the results which can be obtained from Table 1 of Ref. 14 . Thus for the five intervals of $\Delta z=100$ from $z=0$ to $z=500$, we find.

(a): for $z=1$ to $z=100: \quad N_{1}=12, N_{2}=4$ (for $z=65$ and 85), giving $N_{1}+N_{2}$ $=16$,

(b): for $z=101$ to $z=200: N_{1}=12, N_{2}=4$, giving $N_{1}+N_{2}=16$,

(c): for $z=201$ to $300: N_{1}=9, N_{2}=6$, giving $N_{1}+N_{2}=15$,

(d): for $z=301$ to 400 . $N_{1}=8, N_{2}=8$, so that $N_{1}+N_{2}=16$,

(e): for $z=401$ to 500. $N_{1}=7, N_{2}=10$, so that $N_{1}+N_{2}=17$.

Obviously $N_{1}$ decreases from $N_{1}=12$ to $N_{1}=7$ in going from interval (a) to interval (e), whereas $N_{2}$ increases from $N_{2}=4$ to $N_{2}=10$ in going from (a) to (e).

In any case, the near-constancy of $N_{P S}$ (for $\Delta z=100$ ) as a function of $z$ from -100 to $\mathrm{z} \cong 10^{7}$ is a remarkable result, which was directly deduced from the data of Refs. 14 and 16 .

I now wish to discuss two additional papers of mine, namely: (1) "Some Results Involving the Function $\mathrm{n}_{d}$ for Pythagorean Triplets", (Ref. 18); (2) "Some Remarks Concerning the Gregorian Calendar. I" which has already been published.

(Ref. 19).

(1) The first paper (Ref. 18) discusses the values of $n_{d}$ for an integer $X_{1}$, defined by

$$
\mathrm{x}_{1}=\sum_{\mathrm{i}=1}^{\mathrm{n}} \quad \mathrm{p}_{\mathrm{i}}=\mathrm{p}_{1} \mathrm{p}_{2}^{\mathrm{p}_{3}}
$$

where the function $\underset{i=1}{=} p_{i}$ on the right-hand side obviously has the same meaning as in Eq. (17) above; in particular the ordering of the brackets is from the top of the ladder $\left(p_{n}\right)$ 
down to $p_{1}$. As implied in Eq. (69), all of the $p_{i}$ are P-primes. Now it is easy to show that

$$
\mathrm{n}_{\mathrm{d}}\left(\mathrm{x}_{1}\right)=\sum_{\mathrm{i}=2}^{n} \mathrm{p}_{\mathrm{i}}=\mathrm{p}_{2} \mathrm{p}_{3}{ }^{\mathrm{p}_{\mathrm{n}}},
$$

since $X_{1}$ can be written as

$$
\mathrm{x}_{1}=\mathrm{p}_{1}^{\alpha_{1}}, \quad \mathrm{p}_{\mathrm{n}}
$$

where $\alpha_{1}$ denotes the rest of the ladder, i.e. $\alpha_{1}=p_{2} p_{3} \cdot$.

Now we san also consider repeated application of the operator $n_{\mathfrak{d}}$. For example: $\mathrm{n}_{\mathrm{d}}{ }^{2}$ is defined as follows:

$$
\mathrm{n}_{\mathrm{d}}{ }^{2}\left(\mathrm{x}_{1}\right) \equiv \mathrm{n}_{\mathrm{d}}\left[\mathrm{n}_{\mathrm{d}}\left(\mathrm{x}_{1}\right)\right]=\mathrm{n}_{\mathrm{d}}\left(\underset{\mathrm{i}=2}{\stackrel{\mathrm{m}}{=}} \mathrm{p}_{\mathrm{i}}\right)=\underset{\mathrm{i}=3}{\stackrel{\mathrm{n}}{\rightarrow}} \mathrm{p}_{\mathrm{i}}
$$

This powering operation for $n_{d}$ can be repeated several times (depending on $n$ ). It is obvious that we obtain

$$
\begin{gathered}
n_{d}{ }^{n-1}\left(X_{1}\right)=p_{n}, \\
n_{d}{ }^{n}\left(X_{1}\right)=n_{d}\left(p_{n}\right)=1, \\
n_{d}{ }^{n+1}\left(X_{1}\right)=n_{d}(1)=0 .
\end{gathered}
$$

Note that some of the $p_{i}$ can be equal without changing the results of Eqs. (73)(75), e.g. for $n=3$, we may consider

$$
\begin{gathered}
x_{2} \equiv 5^{13^{5}}=5^{371,293} \\
n_{d}\left(x_{2}\right)=13^{5}=371,293 \\
n_{d}{ }^{2}\left(x_{2}\right)=5 \\
n_{d}{ }^{3}\left(x_{2}\right)=1, n_{d}{ }^{4}\left(x_{2}\right)=0
\end{gathered}
$$

so that we still obtain $n_{d}{ }^{n}\left(X_{2}\right)=1(n=3)$, as given by Eq. (79).

However, if one of the $p_{i}$ is replaced by an NP prime $q_{j}[\equiv 3(\bmod 4)]$ or by 2 , the number of $n_{d}$ operations needed to obtain $n_{d}{ }^{v}(X)=0$ is found for $v<n+1$. As an example, I consider

$$
x_{3}=5^{3^{5}}=5^{243}
$$

so that

$$
n_{d}\left(X_{3}\right)=3^{5}
$$




$$
n_{d}{ }^{2}\left(X_{3}\right)=n_{d}\left(3^{5}\right)=0 .
$$

In this case the power $v$ of $n_{d}$ for which $n_{d}\left(x_{3}\right)=0$ is $v=2$ as compared to $v=4$ for the integer $X_{2}$ [see Eq. (79)].

The repeated application of $n_{d}$, i.e., $n_{d}{ }_{n}^{n}$ as defined above has led me to define an index $v$ for any number of the form of $x_{1}={\underset{i=1}{n}}_{i} p_{i}$ or of the type of $x_{3}$, which contains one (or more) NP primes $q_{j}$. [In the present case, i.e., for Eq. (80), $g_{j}=3$ ]. The index $v$ is defined to be that power of $n_{d}$, namely $n_{d}{ }^{v}$ for which $n_{d} v(X)=0$. Obviously $v=n+1$ for a ladder of the type of Eq. (69), e.g., $v=4$ for $X_{2}$ [i.q. (79)].

It occurred to me that the powering of $n_{d}$ and the resulting integers $n_{d}{ }^{k}(X)$ are somewhat analagous to a repeated logarithm-taking. For example:

$$
\ln ^{2} 1000=\ln [\ln 1000]=\ln 6.9078=1.9326 .
$$

We can take two more logarithms, i.e.,

$$
\begin{gathered}
\ln ^{3} 1000=\ln \left[\ln ^{2} 1000\right]=\ln 1.9326=0.6589, \\
\ln ^{4} 1000=\ln 0.6589=-0.4172 .
\end{gathered}
$$

However $\ln ^{\star}(-0.4172)$ does not exist (or more accurately it is a complex number).

Thus we can also define an index $\mu$ for repeated logarithm taking. $\mu$ can be defined as follows: It is the integer $\mu$ for which $\ln ^{\mu} X$ lies in the range $0 \leq \ln ^{\mu} X<1$. Thus for $X=1000$, the logarithmic index is $\mu=3$. In order to determine the value of $\mu$ for an arbritary number $X$ more directly, we calculate the following quantities.

$e=2.71828, e^{e}=15,15426, e^{e^{e}}=3,814,274 \%, e^{e^{e^{e}}}=e^{3,814,274}=10^{1,656,518}$.

Now it is obvious that for $\mathrm{Y} \equiv \mathrm{e}^{\left(\mathrm{e}^{\mathrm{e}}\right)}$, we find

$$
\ln y=e^{e}, \ln ^{2} y=e, \ln ^{3} y=1, \ln ^{4} y=0 \text {. }
$$

Thus for $\mathrm{Y}$ (which can also be written as ${ }^{3} \mathrm{e}$, according to a possible notation), we find that $\mu=4$. In general for a number lying between ${ }^{n}$ and ${ }^{n+1} e$, we find $\mu=n+1$. As an example, since 1000 lies between $e^{e}={ }^{2} e$ and $e^{e^{e}}={ }^{3} e$, we have: 


$$
\mu=n+1=2+1=3 \text {. }
$$

Obviously, an important difference between the functions $n_{d}{ }^{\nu}(X)$ and $\ln ^{\mu} X$ is that $n_{d}{ }^{v}(X)$ is defined only for integer $X$, whereas the logarithmic function $\ln ^{\mu} X$ is defined for all $X$, i.e., it is a continuous function of $X$.

In Ref. 18. I have also given some examples of $v$ and $\mu$ for larger numbers $x_{i}$. It may also be noted that the quantities $e, e^{e}={ }^{2} e,{ }^{3} e$, and ${ }^{4} e$, as given in Eq. (86) determine a natarai scale for very large numbers. Obviously $5_{\mathrm{e}}=\mathrm{e}^{10^{1,656,518}}$ is "astronomically" large.

(2). Concerning Ref. 19 on properties of the Gregorian Calendar, I will give only a brief description, since this paper has been recently published in Jour. Recr. Math, vol. 21, p.91-96 (1989). This paper contains results on two main topics: (a) the deviations from $1 / 7$ for the probability that a particular day of the year, e.g. January 1, be a Thursday (or any other day of the week). As was suggested to me by Elliot Auertach, these deviations occur as a result of the fact that for the Gregorian Calendar (as opposed to the Julian Calendar), the century-years when the year is not divisible by 4 are not leap years. (e.g., 1700, 1800, 1900, 2100). As an example, for July 15. the probabilities $\mathrm{P}$ (Sunday), $\mathrm{P}$ (Monday), etc. are given by

$\mathrm{P}($ Sunday $)=58 / 400 ; \mathrm{P}($ Monday $)=57 / 400 ; \mathrm{P}($ Tuesday $)=57 / 400 ; \mathrm{P}($ Wednesday $)=58 / 400$;

$$
P(\text { Thursday })=56 / 400, P(\text { Frida } y)=58 / 400, P(\text { Saturday })=56 / 400 \text {. }
$$

The three values $56 / 400=0.1400,57 / 400=0.1425,58 / 400=0.1450$ are close to but obviously not exactly equal to $1 / 7=0.14286 \ldots$ From the Eqs. (89), the probabilities; $\mathrm{P}$ (Sunday), $\mathrm{P}$ (Monday),...etc. can be determined for any day of the year, except for February 29 in the case of leap years. For February 29, a separate calculation gave three possible values of the probabilities; $\mathrm{P}$ (Sunday), $\mathrm{P}$ (Monday), etc. These 3 values (analogous to $56 / 400,57 / 400$ and $58 / 400$ above) are: $13 / 97=0.134, \quad 14 / 97=0.144$, and 15/ 97=0.155. An interesting result is that if we take all February 29's of a 400-year cycle, the probability that February 29 be on a Monday divided by the probability that the same date occurs on a Sunday is 


$$
\frac{P_{29} \text { (Monday) }}{P_{29} \text { (Sunday) }}=\frac{15}{13}=1.154 \text {. }
$$

(b) The second result of the paper concerns a device to determine the day of the week for a particular date, say July 4, 1942, from the day of the week for the same date, of a different year, using the two natural cycles of the Gregorian Calendar, namely the two periodicities of 28 years and 400 years, respectively. For details, the reader is referred to my paper (Ref. 19). Additional results concerning the Gregorian Calendar are published in Refs. 5, 6 and 20.

Finally I want to discuss some very recent results on the system of prime numbers which I have submitted to J. Recr. Math. in August 1989 (BNL publication, BNL-43,227), (see Ref. 21). In connection with the coming decade, 1990-2000, I noticed that there are no less than 3 prime numbers in this relatively small interval, namely: 1993, 1997, and 1999. Obviously the last two numbers (1997, 1999) represent a set of twin primes. In view of the fact that the 10 other primes in the interval from 1900 to 2000 are spaced rather far apart (with the exception of the two twin sets, 1931, 1933 and 1949, 1951), I came to the tentative conclusion that what I call prime-triplets (3 primes within a decade, like 1993, 1997, 1999) are relatively rare. For this reason, I made a systematic study and listing of all prime triplets in the range from 1 to 10,000 .

When I started to study this problem, I noticed that there are also sets of primequadruplets (four primes within a decade), and although these are less frequent than the sets of prime-triplets, neither of these two sets are extremely rare. As a matter of fact the interval from 1870 to 1880 contains the prime-quadruplet; $1871,1873,1877$, and 1879. A necessary, but by no means sufficient condition for the possibility of a primequadruplet is that the central odd number (1875 in the present case) i.e., the one which is divisible by 5 must also be divisible by 3 , i.e., it must be divisible by 15 .

In the interval from $\mathrm{N}=1$ to $\mathrm{N}=3000$, there are only 8 prime-quadruplets, namely 1,3,5,7 (which should also include $N=2$, thus making it the only prime- quintuplet), 
$11,13,17,19 ; 101,103,107,109 ; 191,193,197,199 ; 821,823,827,829 ; 1481,1483,1487.1489$;

1871,1873,1877,1879; and 2081,2083,2087,2089. In Table 1 of my paper; (Ref.21), I have made a complete listing of all triplets and quadruplets in the range from $N=1$ to $\mathrm{N}=10,000$. There are altogether 63 prime-triplets and 12 prime-quadruplets in this range, involving a total of 237 prime numbers.

In Table 2 of this paper (Ref. 21 ), I have obtained the individual numbers $N\left(Y_{1}, y_{2}\right)$ of prime numbers associated with the triplets and quadruplets, and the total number of primes $\pi\left(Y_{1}, Y_{2}\right)$ in the same range $\left(Y_{1}, t_{2}^{Y_{2}}\right)$, and the corresponding ratios $R\left(Y_{1}, Y_{2} 1 / Z\right)$, defined as.

$$
R\left(Y_{1}, \frac{Y_{2}}{7}\right) \equiv N\left(Y_{1}, \frac{1}{2}\right) / \pi\left(Y_{1}, \frac{1 / 2}{2}\right) \text {. }
$$

As expected, the ratio $R\left(Y_{1}\right.$, decreases on the whole with increasing $Y$, starting with $R(Y)=0.30$ for $Y \approx 1000$, to $R(Y) \approx 0.154$ for $Y=8750$. Here $Y$ is a suitable average of the end points $Y_{1}, Y_{2}$, i.e., $Y=\left(1 / 2 / Y_{1}+1 / 2\right)$. In a similar manner to my earlier papers (Refs. 14 and 16), I have fitted $R(Y)$ to a function of the form $R(Y)=A Y^{-\alpha}$. The result is:

$$
R(Y)=1.191 \mathrm{Y}^{-0.1996}=1.191 \mathrm{Y}^{-1 / 5.010} \text {. }
$$

In as much as the symbol $\mathrm{Y}$ (which originally stood for "year") applies generally to the integer $N(=Y)$, Eq. (92) can be rewritten as:

$$
R(N)=1.191 \mathrm{~N}^{-0.1996}=1.191 \mathrm{~N}^{-1 / 5.010} \text {, }
$$

(see Table 3 of Ref. 20).

Equation (93) can be used to give a proof that the total number of prime-triplets and quadruplets for integers $<\mathrm{N}$ becomes infinite as $\mathbf{N} \rightarrow \infty$. For this purpose, I use the very accurate formula of Legendre for the number $\pi(N)$ of primes $\leq N$. This formula is:

$$
\pi(\mathrm{N})=\frac{\mathrm{N}}{\ln \mathrm{N}-1.0836} \text {. }
$$

From Eq. (94), one finds that the number of primes in a suitably small interval near $N$, i.e., from $N-\frac{\Delta N}{2}$ to $N+\frac{\Delta N}{2}$, is approximately given by

$$
\Delta \pi(\mathrm{N})=\frac{\Delta \mathrm{N}}{\ln \mathrm{N}-1.0836}
$$

For large $N$ (essentially for $\left.N^{>} e^{10}=22,026\right)$, we find: 


$$
\mathrm{d} \pi(\mathrm{N}) \approx \frac{\mathrm{dN}}{\ln N}
$$

Now from Eq. (93), we have

$$
R \approx 1.19 N^{-Q 2} \text {, }
$$

and therefore the number of primes involved in the prime triplets and quadruplets category (TQ) is given by

$$
\pi_{\mathrm{TQ}}\left(\mathrm{N}_{0}\right) \approx \int_{0}^{\mathrm{N}_{0}} \frac{1.19 \mathrm{~N}^{-0.2 \mathrm{dN}}}{\ln \mathrm{N}}
$$

Obviously this integral diverges for large $N_{0}$, as can then be seen by writing: $\ln _{-} N=X, N=e^{X}$, so that $d N=e^{x} d X, N^{-02}=e^{-0.2 X}$. This gives:

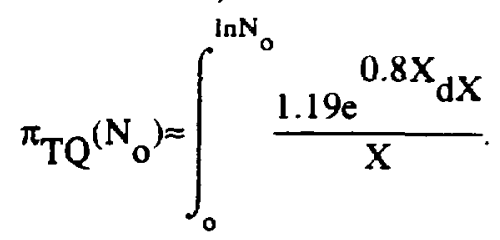

Since the exponential in the numerator $e^{0.8 X}$ has a positive coefficient of $X$, the integral diverges for large $N_{0}$, i.e., for large $X$. The preceding argument [Eqs. (94)(99)] provides a proof that the number of criplet and quadruplet primes is infinite.

A completely similar proof can be given for the total number of twin primes $<N_{0}$, i.e., $\pi_{T W}\left(N_{0}\right)$, for which the integral corresponding to Eq. (99) also diverges for large $\mathbf{N}_{\mathbf{o}}$. In this connection, I refer the reader to my paper of Ref. 18 (BNL-41165).

In this paper, I have discussed (at the end of the paper) the ratio $R_{T W}$ of the number $\mathbf{N}_{\text {TW }}$ of twin primes to the total number of primes, i.e.,

$$
\mathbf{R}_{\text {TW }}=\frac{\mathbf{N}_{\text {TW }}}{\mathbf{N}_{\text {all primes }}},
$$

in a small interval $\Delta \mathrm{N}$ from $\mathrm{N}-\frac{\Delta \mathrm{N}}{2}$ to $\mathrm{N}+\frac{\Delta \mathrm{N}}{2}$. I used the same numerical results as in Ref. 16, i.e., the tables of prime decompositions obtained by Dr. M. Creutz.

$R_{T W}$ decreases slowly with increasing $N$, in a similar manner to the behavior of $R_{T Q}$ (see Table 3 of Ref. 18). As examples, $R_{T W}(1850)=0.424$ (for the integers near $\mathrm{N}=1850), \mathrm{R}_{\mathrm{TW}}(22,050)=0.286$, and $\mathrm{R}_{\mathrm{TW}}\left(10^{7}\right)=0.179$. Upon fitting these values (re- 
quired by obtaining exact agreement at $\mathrm{N}=1850$ and $\mathrm{N}=10^{7}$ ), I have obtained the following approximate formula (see Ref. 18):

$$
R_{T W}(\mathrm{~N})=0.902 \mathrm{~N}^{-0.1003}=0.902 \mathrm{~N}^{-1 / 9.97} \text {. }
$$

Thus $R_{T W}(N)$ decreases less fast with increasing $N$ (exponent $=0.1003$ ) than the ratio $R(N)$ obtained above for the triplet and quadruplet primes (exponent $=0.1996$ ). Hence the argument given above for $\pi_{\mathrm{TQ}}\left(\mathrm{N}_{\mathrm{o}}\right)$ [see Eq. (98)] holds in the same way. Upon writing $X=\ln N, N=e^{X}$, we obtain [instead of Eq. (99)].

$$
\pi_{\mathrm{TW}}\left(\mathrm{N}_{\mathrm{o}}\right)=\int_{0}^{\ln \mathrm{N}_{\mathrm{o}}} \frac{0.902 \mathrm{e}^{0.9 \mathrm{X}} \mathrm{dX}}{\mathrm{X}}
$$

which obviously diverges as $\mathrm{N}_{0}$ becomes very large.

It is interesting to note that the exponent of $N$ for $R_{T W}(i . e .,-0.1003)$ is almost exactly one-half the exponent of $\mathrm{N}$ which I have previously obtained for the triplet-quadruplet primes (i.e.,-0.1996). This can also be understood in terms of the distribution of prime numbers, since it is relatively more likely to obtain a set of twin primes at large $\mathrm{N}$, rather than a set of triplets or quadruplets. Nevertheless in both cases the exponents of $N$ for $R\left(=R_{T Q}\right)$ and $R_{T W}$ are negative and well below 1 , so that the exponents of $e$ in Eqs. (99) and (102) are both positive $\left(e^{0.8 X}\right.$ and $\left.e^{0.9 X}\right)$, which ensures that $\pi_{\mathrm{TW}}(\mathrm{N})$ and $\pi_{\mathrm{TQ}}(\mathrm{N})$ increase indefinitely with increasing $N$, so that the total number of the twin primes and of the triplet-quadruplet primes are both infinite.

In addition, I have also recently made a study of the palindromic integers, i.e., integers which "read" the same forward and backward. Examples are: 131, 868, $1441,8558,12521,125,2521$, etc. The details will be published in Ref. 22 . The palindromes are of the form abba when the number of digits $n_{D}=4$, and abcba, when $n_{D}=5$. Besides obtaining the total number of palindromes 
$\sum_{1}^{N} N_{\text {pal }}$ (all) less than any value of $N$ of the form $N=10^{a}$, where a goes from $a=1$ to $a=11$ [see Table 1 of Ref. 22], I also noticed and proved that when the number of digits $\mathrm{n}_{\mathrm{D}}$ is even, all of the palindromes $\mathbf{N}_{\text {pal }}$ are divisible by 11 . As examples, 1441/ $11=131$, $168861 / 11=15351$. However when $n_{D}$ is odd as for $n_{D}=3$, only approximately $1 / 11$ of the total number of palindromes $\mathbf{N}_{\text {pal }}$ is divisible by 11 , as would be expected for a random set of integers $\mathbf{N}$. In addition, of those palindromes which are divisible by 11 , an appreciable fraction is divisible by $11^{2}=121$ or even by $11^{3}=1331$ and $11^{4}=14,641$. For this reason, I have constructed 41 palindromes by considering all integers of the form $N_{n}=N_{1}(11)^{n-1}$, where $N_{1}$ consists of 0 's and l's only, and the number of digits of $N_{1}$, namely $n_{D}\left(N_{1}\right)$ takes on the values $2,3,4,5$, and 6 . The results are given in Table 2 of Ref. 22.

In addition to my work on palindromes (Ref. 22), I also wrote up some work which I had done earlier, namely an investigation of the properties of the function $E(a b c)$ or E(abcd), where

$$
E(a b c) \equiv a^{b^{c}} ; E(a b c d) \equiv a^{b^{c}}
$$

where (abc) are the 3 digits of an arbritary integer with 3 digits, and similarly for ${ }^{n}=4$, i.e. the number (abcd). The details of this work are given in Ref. 23. Incidentally, by $E=a^{b^{c}}, I$ mean

$$
E(a b c) \equiv a^{\left(b^{c}\right)}
$$

i.e., the bracketing must start at the top of the ladder and going down. In the same manner,

$$
\left.E(a b c d) \equiv b^{\left[b^{()}\right.}\right] .
$$

In my paper (Ref. 23), I have shown that if none of the integers $a, b, c, d$ are either 0 or 1, then one can obtain huge numbers. Thus, as an example,

$$
E(5555)=5^{5^{5}}=5^{5^{3125}}=5^{10^{2184.2813}}=10^{10^{2184.1258}},
$$

which is a huge number since it has $10^{2184}+1$ digits. 
On the other hand, if one of the integers abcd $\mathrm{E}$ (abcd) is 0 or 1 , the value of $E(a b c d)$ is quite small. As examples:

$$
E(2817)=2^{8^{1^{7}}}=2^{8}=256 ; E(25010)=2^{5^{0^{0}}}=2^{5^{0}}=2^{1}=2 \text {. }
$$

For further details, the reader is referred to Ref. 23.

Finally, the paper entitled "Additional Remarks Concerning the Gregorian Calendar" (Ref.20), contains results for $D_{1}(Y)$ defined as the day of the week of January 1 of the year $Y$. The principal results are contained in Tables 1 and 2 of this paper. Table 1 lists the results for $D_{1}(Y)$, when $Y=1900,1910, \ldots 1990,2000$, followed by the values of $D_{1}(Y)$ for the century-years from $Y=2000$ to $Y=10,000$. In general, for a sequence of 5 consecutive century-years, e.g., 2000,2100,2200,2300, 2400 we have the sequence: Saturday $\left[=D_{1}(2000)\right]$, Friday, Wednesday, Monday and Saturday, respectively, partly because the Gregorian Calendar has a natural period of 400 years after which the calendar repeats itself, i.e.,

$$
D(\text { Day, } Y)=D(\text { Day, } Y+400 \text { years }) \text {, }
$$

where $D($ Day, $Y)$ denotes the day of the week for any particular day of the year $Y$.

Table 2 of the paper (Ref. 20) gives the results for $D_{1}(Y)$ for the years $1,100,200$, $300, \ldots 1800,1900$, and 2000 in steps of 100 (except for the interval from 1 A.D. to $100 \mathrm{~A}$. D.). In the last part of the paper, I have determined the number of days $\mathrm{T}$ which have elapsed from Sunday, January 1, 1 A.D. to Monday, January 1, 1990. This interval $T=726,468$ days. The contribution to $T$ from the time that the Gregorian Calendar was introduced, namely Friday October 15, 1582 in Italy (and the other Catholic countries of Western Europe, i.e., France, Spain and Portugal) is; $T_{2}=148,732$ days. Thus the amount of time during which the Julian Calendar (starting January 1, 1 A.D.) was in effect is: $\quad T-T_{2}=726,468-148,732=577,736$ days. 


\section{ACKNOWLEDGMENTS}

I would like to thank Dr. Michael Creutz for many helpful discussions in connection with several aspects of this work, and in particular, for his helpful collaboration on our 4 papers of Refs. 7, 10, 11, and 12, and for several calculations carried out on his IBM-PC Computer. I am also very much indebted to Dr. Elliot Auerbach, Dr. Sidney Heller, Dr. Joseph S. Madachy, and Mr. Bruce Martin for valuable suggestions and comments.

Last but not least, I wish to thank my wife, Elizabeth P. Sternheimer, for her patient and most helpful encouragement and her constant moral support, without which my present research on number theory would not have been possible.

The present work was supported by the U.S. Department of Energy under Contract No. DE-AC02-76CH00016.

\section{REFERENCES}

1. R.M. Sternheimer, "Shielding and Antishielding of Nuclear Quadruphe Moments", Zeerscirift fin Nitufinchang 41 a, 24-36 (1986).

2. R.M. Sternheimer, Phys. Rev. 88,851 (1952).

3. R.M. Stemheimer, M.J. Berger and S.M.Seltzer, Atomic Data and Nuclear Data Tables, 30, 261-271 (1984).

4. L. Euler, "Introduction in Analysis Infinitorum" (1748); quoted by R.A. Kynoebel, "Exponentials Reiterated", American Math. Monthly, 요, 235 (1981); see also the preprint (1977).

5. R.M. Sternheimer, "Some Remarks Concerning the Gregorian Calendar, II". Journal of Recreational Mathematics, 22, 39-51 (1990).

6. R.M. Sternheimer, "Some Remarks Conceming the Gregorian Calendar III", BNL42903, to be published in J. Recr. Math., vol 22 (1990). 
20. R.M. Sternheimer, "Additional Remarks Concerning the Gregorian Calendar", Report BNL-44439, submitted to J. Recr. Math. (March 1990).

21. R.M. Sternheimer, "Properties of the Prime-Number Triplets and Quadruplets", Report BNL-43227, J. Recr. Math., to be published in Vol. 22 (1990).

22. R.M. Sternheimer, "On Palindromic Numbers and Their Divisibility by 11", Report BNL-44387, submitted to J. Recr. Math. (February 1990).

23. R.M. Sternheimer, "On Certain Integers which Are Obtained by Repeated Exponentiation", submitted to J. Recr. Math. (January 1990), Report BNL-44349. See also: R.M. Sternheimer, "On a Result Involving Iterated Exponentiation", Fibonacci Quarterly, 26, 178-180 (1988). 
7. M. Creutz and R.M. Sternheimer, "On a Class of Non-Associative Functions of a Single Positive Real Variable", BNL-23308 (September 1977); see Appendix A by R.M.S., pages $27-30$ and Table $V$ on page 35 .

8. R.M. Sternheimer, "The k-Ordering of Atomic Structure" in Progress in Atomic Spectroscopy, Part C, pp. 1-27 (Plenum Press, New York, 1984). See also my original papers: R.M. Sternheimer, Phys. Rev. A $\underline{15}, 1817$ (1977); A $\underline{16}, 459$ (1977); A 16, 1752 (1977); A 19, 474 (1979); and A 20, 18 (1979).

9. R.M. Stemheimer, "Cn a Set of Non-Associative Functions of a Single Positive Real Variable", BNL Report, BNL-23081 (June 1977).

10. M. Creutz and R.M. Sternheimer, Fibonacci Quarterly 18, 341-347 (1980).

11. M. Creutz and R.M. Sternheimer, Fibonacci Quarterly 19, 326-335 (1981).

12. M. Creutz and R.M. Stemheimer, Fibonacci Quarterly 20, 7-12 (1982).

13. R.M. Sternheimer, "A Corollary of Iterated Exponentiation", Fibonacci Quarterly 23, $146-148(1985)$.

14. R.M. Sternheimer, Fibonacci Quarterly 24, 107-128 (1986).

15. E.J. Eckert, Mathematics Magazine, 57, 22-27 (1984).

16. R.M. Sternheimer, "Some Results Conceming Pythagorean Triplets, II", Jour. Recr. Math. 21, 264-275 (1989).

17. R.M. Stemheimer, "Some Remarks Concerning the Frequency of the Primitive Solutions of the Pythagorean Equation: $x^{2}+y^{2}=z^{2}$ ", Report BNL-41569, submitted to J. Recr. Math. (July 1988).

18. R.M. Sternheimer, "Some Results Involving the Function $n_{d}$ for Pythagorean Triplets", Report BNL-41165, submitted to J. Recr. Math. (March 1988).

19. R.M. Sternheimer, "Some Remarks Concerning the Gregorian Calendar", J. Recr. Math. 21, 91-96 (1989). 